\title{
COMMUTATIVITY OF UNBOUNDED NORMAL AND SELF-ADJOINT OPERATORS AND APPLICATIONS
}

\author{
MOHAMMED Hichem MORTAD
}

Abstract. Devinatz, Nussbaum and von Neumann established some important results on the strong commutativity of self-adjoint and normal unbounded operators. In this paper, we prove results in the same spirit.

Mathematics subject classification (2010): Primary 47A05; Secondary 47B25.

Keywords and phrases: Unbounded selfadjoint and normal operators, invertible unbounded operators, operator products, strong commutativity.

\section{REFERENCES}

[1] A. ARAI, Generalized weak Weyl relation and decay of quantum dynamics, Rev. Math. Phys., 17/9 (2005), 1071-1109.

[2] S. M. Barnett, P. M. Radmore, Methods in Theoretical Quantum Optics, Oxford 1997.

[3] J. B. CONwAY, A Course in Functional Analysis, Springer, 1990 (2nd edition).

[4] A. Devinatz, A. E. Nussbaum, J. Von Neumann, On the Permutability of Self-adjoint Operators, Ann. of Math. (2), 62 (1955), 199-203.

[5] A. Devinatz, A. E. Nussbaum, On the Permutability of Normal Operators, Ann. of Math. (2), 65 (1957), 144-152.

[6] B. Fuglede, A Commutativity Theorem for Normal Operators, Proc. Nati. Acad. Sci., 36 (1950), 35-40.

[7] B. Fuglede, Conditions for Two Selfadjoint Operators to Commute or to Satisfy the Weyl Relation, Math. Scand., 51/1 (1982), 163-178.

[8] F. R. Gantmaher, M. G. KREIn, O normalnyh operatorah v ermitovom prostranstve [Russian], Izv. fiz-mat ob-vapri Kazanskom Univ., 1/3 (1929-1930), 71-84.

[9] F. Gesztesy, J. A. Goldstein, H. Holden, G. Teschl, Abstract Wave Equations and Associated Dirac-Type Operators, Annali di Matematica. DOI 10.1007/s10231-011-0200-7.

[10] A. GheondeA, When Are the Products of Normal Operators Normal? Bull. Math. Soc. Sci. Math. Roumanie (N.S.) 52(100)/2 (2009), 129-150.

[11] I. Gohberg, S. Goldberg, M. A. KaAshoek, Basic Classes of Linear Operators, Birkhäuser Verlag, Basel, 2003.

[12] P. E. T. Jorgensen, Unbounded Operators: Perturbations and Commutativity Problems, J. Funct. Anal. 39/3 (1980), 281-307.

[13] I. KaPlansky, Products of Normal Operators, Duke Math. J., 20/2 (1953), 257-260.

[14] M. H. Mortad, An Application of the Putnam-Fuglede Theorem to Normal Products of Selfadjoint Operators, Proc. Amer. Math. Soc. 131 (2003), 3135-3141.

[15] M. H. Mortad, On Some Product of Two Unbounded Self-adjoint Operators, Integral Equations Operator Theory, 64 (2009), 399-408.

[16] M. H. Mortad, On the Closedness, the Self-adjointness and the Normality of the Product of Two Unbounded Operators, Demonstratio Math., 45/1 (2012), 161-167.

[17] M. H. Mortad, On the Normality of the Sum of Two Normal Operators, Complex Anal. Oper. Theory, 6/1 (2012), 105-112. DOI: 10.1007/s11785-010-0072-7.

[18] M. H. Mortad, On the Normality of the Unbounded Product of Two Normal Operators, Concrete Operators, 1 (2012), 11-18. DOI: 10.2478/conop-2012-0002. 
[19] E. Nelson, Analytic Vectors, Ann. of Math. (2), 70 (1959), 572-615.

[20] A. E. Nussbaum, A Commutativity Theorem for Unbounded Operators in Hilbert Space, Trans. Amer. Math. Soc. 140 (1969), 485-491.

[21] A. E. Nussbaum, A Commutativity Theorem for Semibounded Operators in Hilbert Space, Proc. Amer. Math. Soc. 125/12 (1997), 3541-3545.

[22] C. R. Putnam, On Normal Operators in Hilbert Space, Amer. J. Math., 73 (1951), 357-362.

[23] M. Reed, B. Simon, Functional Analysis, Methods of Modern Mathematical Physics, Vol. 1, Academic Press, 1972.

[24] W. Rudin, Functional Analysis, McGraw-Hill, 1991 (2nd edition).

[25] K. SchmÜDgen, J. Friedrich, On Commuting Unbounded Selfadjoint Operators II, Integral Equations Operator Theory, 7/6 (1984), 815-867.

[26] K. SCHMÜDgen, Strongly Commuting Unbounded Selfadjoint Operators and Commutants of Unbounded Operator Algebra, Proc. Amer. Math. Soc. 102/2 (1988), 365-372.

[27] K. SchmÜDgEn, Unbounded Self-adjoint Operators on Hilbert Space, Springer GTM 265, (2012).

[28] Z. Sebestyén, J. Stochel, On Products of Unbounded Operators, Acta Math. Hungar., 100/1-2 (2003), 105-129.

[29] T. Tudor, A. Gheondea, Pauli Algebraic Forms of Normal and Non-Normal Operators, J. Opt. Soc. Am. A, 24 (2007), 204-210.

[30] J. WeIDmAnN, Linear operators in Hilbert spaces (translated from the German by J. Szücs), SrpingerVerlag, GTM 68 (1980).

[31] N. A. Wiegmann, Normal Products of Matrices, Duke Math J., 15, (1948), 633-638.

[32] N. A. Wiegmann, A Note on Infinite Normal Matrices, Duke Math. J., 16 (1949), 535-538.

[33] S. M. Zagorodnyuk, On commuting Symmetric Operators, Methods of Functional Anlaysis and Topology, 18/2 (2012), 198-200. 